\title{
The correctness and relevance of the modal ontological argument
}

\section{Andrzej Biłat ${ }^{1}$ (1)}

Received: 12 May 2020 / Accepted: 8 October 2020/Published online: 21 December 2020

(C) The Author(s) 2020

\begin{abstract}
This paper deals with some metaphilosophical aspects of the modal ontological argument originating from Charles Hartshorne. One of the specific premises of the argument expresses the idea that the existence of God is not contingent. Several well-known versions of the argument have been formulated that appeal to different ways of clarifying the latter. A question arises: which of the formally correct and relevant versions is proper or basic? The paper points to some criteria of formal correctness, and distinguishes two types of relevance for these versions: strong and weak. Its aim is to furnish a strictly worked out answer to the question, taking into account each of these types. As a result, a very simple, formally correct and (weakly) relevant version of the modal ontological argument is formulated. The results obtained are also used to criticize a popular belief about the relations in which the main versions of the modal ontological argument stand to one another.
\end{abstract}

Keywords Structure of argument · Formal correctness of argument - Simplicity of argument $\cdot$ Relevance of argument $\cdot$ Modal logic $\cdot$ Mini-theories

of God

\section{Introduction}

Ontological arguments amount to a priori arguments for philosophical theism: i.e. the thesis that God, in a philosophical sense of the word, exists. There are many (at least seven) types of such arguments (Oppy 2019). One of them is the modal ontological argument (hereinafter MOA), an argument formalizable in a simple

Andrzej Biłat

andrzej.bilat@pw.edu.pl

1 Faculty of Administration and Social Sciences, Warsaw University of Technology, Warsaw, Poland 
zero-order language of (applied) modal logic or an (appropriately enriched) standard first-order language of the theory of possible worlds. ${ }^{1}$

More particularly, in the form in which it originated in the work of Charles Hartshorne, the MOA is formulated within a zero-order modal theory, in which the only extra logical constant is the sentence "God exists". This argument is based on two specific metaphysical premises. The first of these states that the existence of God is possible. This premise is nowadays most often considered to be the core of the MOA. ${ }^{2}$ The second premise is an explication of the idea that the existence of God is not contingent (Hartshorne 1944). Depending on the way in which this idea is explicated, different versions of the MOA come to be formulated.

More complex versions, coming from Alvin Plantinga, are formulated within a standard first-order theory of possible worlds extended by the concept of God (Plantinga 1974). ${ }^{3}$ In this paper we will confine ourselves to just the Hartshornestyle zero-order MOA-versions. (For convenience, we will continue to use the phrases "Hartshorne-style argument" and "MOA" interchangeably.) ${ }^{4}$

One of the simpler (zero-order) MOA-versions (though not necessarily the simplest-see the next footnote and Sect. 4 below) is presented in the Stanford Encyclopedia of Philosophy as follows:

It is possible that God exists. God is not a contingent being, i.e., either it is not possible that God exists, or it is necessary that God exists. Hence, it is necessary that God exists. Hence, God exists. ${ }^{5}$

Despite its formal simplicity, arguments of this type are still the subject of numerous analyses. In particular, their persuasive power is studied, and often also questioned (see Oppy 1996, 2019). We do not, however, intend to address that issue here (except for the analysis of the persuasive function of one of the MOA versions).

\footnotetext{
1 These versions basically have their origins in Hartshorne (1944), Malcolm (1960) and Plantinga (1974) (although their intuitive formulation already appears in St. Anselm's Proslogion III; see Eder and Ramharter 2015, p. 2815; Oppy 2019). They are clearly distinguished here from arguments of the Gödelian kind formulated within second- or higher-order modal theories of positive properties (see Sobel 1987; Benzmüller 2020). The MOAs and Gödelian-type arguments are also specified in Oppy's taxonomy as two different kinds of ontological argument (Oppy 2019). The abbreviation "MOA" is most commonly used in the philosophical literature to designate the former. (Even so, the term "modal ontological argument" could also be used in the broader sense of "ontological argument using modal concepts", and in this sense, Gödelian-type arguments could also be called "modal".).

${ }^{2}$ G.W. Leibniz is commonly regarded as the philosopher who was the first to point out the key role of this premise in the ontological argument (see, e.g., Perzanowski 1991; Antognazza 2018). The issue of the reliability of this premise will not be discussed in the present paper.

3 The argument presented in Lewis (1970) is an example of another MOA-version formulated within an extensional first-order theory of possible worlds.

4 These versions belong to one of the four kinds of MOA distinguished in Oppy (1996, pp. 70-78). This kind includes arguments of the sort Oppy calls "ontological arguments involving necessity".

5 Oppy (2019). This version was already considered by Hartshorne (see Goodwin 1978). An even simpler MOA-version is analyzed in Oppy (1996): "It is possible that it is necessarily the case that God exists. Hence, God exists." This version is valid on the basis of systems B and S5 of modal logic. Other B- and S5-versions of the MOA will be presented in Sect. 4. (The T, B and $\mathbf{S 5}$ systems are deductive extensions of classical sentential logic, and belong to what is known as normal modal logic. Chellas (1980) offers an accessible characterization of what they involve.).
} 
The main subject of our present study is, rather, the general structure of Hartshornestyle MOAs, the basic properties of their most important versions, and some relations between them. Its main purpose is to distinguish the basic versions from all other MOAs that meet predetermined criteria of formal correctness and relevance.

The following view seems prevalent in the philosophical literature: certain general premises generated by stronger systems of modal logic are logically essential, or at least philosophically the most adequate ones, where the MOA is concerned. ${ }^{6}$ This line of thinking was clearly expressed by philosophers quite early on. For example, Kane (1984) lists the general premises (taken from B-system) as the third important element-after two specific premises-in the construction of the MOA. ${ }^{7}$ In recent years, a similar view has been presented by van Inwagen (2012, 2018) in a more cautious way, clearly suggesting two theses: (1) each formally correct and relevant MOA-version has either strong specific premises and weak general premises (generated by modal logic) or, equally, weak specific premises and strong general premises; (2) the second part of this equivalence is the philosophically preferable one (on account of its clarity and elegance). ${ }^{8}$

In this paper, I will try to show that if we assume some quite natural criteria for the philosophical evaluation and selection of MOA-versions, both of the above theses turn out to be wrong.

The main points of reference here are those normal systems of modal logic that are the most widely known ones (at least in the context of MOA): i.e. systems in which Modus Ponens and the Rule of Necessitation make up the only primitive rules. The purpose of the study is not to analyze possible logical systems underlying various MOA-versions, but rather to analyze these versions on the basis of a predetermined system of modal logic: one that is - in a way-the "internal" logic of every MOA-version. Thus, the determination of a given MOA-version will not be done by changing this "internal" logic (e.g. by adding new logical rules), but by

\footnotetext{
${ }^{6}$ It is worth noting that in recent years an opposite trend has appeared in studies on Gödelian-type of ontological argument (which was originally formulated within the S5-system, cf. Sobel 1987). For example, Świętorzecka and Łyczak (2018) reconstruct a version of this argument within the S4-system, and Benzmüller (2020) within the T-system and even in the weaker K-system. The analyses undertaken in this paper go in a similar direction, that is, towards the search for possibly simple and adequate MOAversions.

7 This view was also adopted in a well-known philosophical dictionary, in which one of the S5-versions was commented on as follows: "The correct response to this argument is to disallow the apparently reasonable concession that it is possible that such a being exist. This concession is much more dangerous than it looks, since in the modal logic involved, from possibly necessarily $p$, we can derive necessarily $p$ " (Blackburn 1994, p. 269). Cf. also the footnote 18.

8 "Here is the version I think is the clearest and most elegant" (van Inwagen 2012, p. 157). "[...] the modal logic of the argument is $\mathbf{S 5}$, the strongest modal system. This is not the case with every version of the modal argument. Some are valid in weaker modal systems, but those arguments require additional premises" (ibid., p. 158). "One could regard the first premise of each of Hartshorne's arguments [equivalent to formula (2)] as substitutes for an appeal to the strong modal system S5" (van Inwagen 2018, p. 242).
} 
changing or adding new premises, including both substantive (metaphysical) and general premises taken from various "external" systems of modal logic. 9

The findings proposed in the next section provide fairly strict answers to two questions: namely, what the overall structure of the (Hartshorne-style) MOA and the criterion for the latter's relevance look like. There are also two other closely related and logically basic questions, which concern what the correctness and simplicity criteria for MOA-versions are, and which of the known correct and relevant versions is the simplest. In the philosophical literature, we do not find precise answers to these questions. Answers to the above questions can then help with solving the key metaphilosophical issue of which MOA-version is proper or basic.

The structure of the ensuing discussion is as follows: the next two sections set out the structure of the MOA and criteria for its formal correctness and simplicity, while Sect. 4 furnishes proofs of the correctness of the T-version, S5-version, and B-version; Sect. 5 then presents a correct, simplified MOA-version, and Sect. 6 distinguishes two types of philosophical relevance for MOA-versions; finally, Sect. 7 uses these findings to criticize a popular view related to van Inwagen's theses (1) and (2).

\section{The structure and relevance of the modal ontological argument}

For the purposes of the present analysis, we shall retain the principle of the possibility of the existence of God (expressed in the first sentence of the quotation presented in the previous section), expressing this with the symbolization " $\mathrm{Mg}$ " and assuming it to be obligatory for every MOA-version:

Taking the symbols "Lg" to represent the sentence "It is necessary that God exists", the second sentence of the above quotation can then be rewritten using the formula:

\footnotetext{
9 Taking into account this assumption, we will omit systems possessing a different set of primitive rules, such as $\mathbf{D}+$ and $\mathbf{T}+($ with the so called MacIntosh rule), which are sometimes also considered in MOArelated literature (see Chellas and Segerberg 1994).

10 The operators M and L can also be understood as formal counterparts of Anselm's "it is conceivable that" and "it is not conceivable that not", respectively (see Eder and Ramharter 2015, p. 2814). In turn, for the constant $\mathrm{g}$, the following interpretation is sometimes proposed: "There is a necessarily existent being that has all perfections essentially" (van Inwagen 2012, p. 158, 2018, p. 242).
} 
The sentence $g$ ("God exists") is derived from the premises (1) and (2), with the use of the law of double negation, disjunctive syllogism (modus tollendo ponens), and the following general (non-specific) premise resulting from the application of the axiom ' $\mathrm{L} \alpha \Rightarrow \alpha$ ' of the $\mathbf{T}$-system of modal logic ${ }^{11}$ :

Considering the above, we may conclude that this argument for (philosophical) theism is a valid (i.e. logically correct) MOA-version that is based on two specific premises, (1) and (2), and one general premise T. ${ }^{12}$

In other MOA-versions, the choice of specific and general premises changes. In typical versions, (2) is weakened and the $\mathbf{T}$-system is strengthened by adding new general premises. ${ }^{13}$ These additional premises are usually taken from the S5-system or, less often, from the weaker B-system.

The $\mathbf{T}$-system is the weakest system of normal modal logic in which the uncontroversial formulae of the form ' $\mathrm{L} \alpha \Rightarrow \alpha$ ' are theses. Regardless of whether or not these formulae are used in a particular MOA-version, it is the basic system for explicating the meaning of the modal operators $\mathrm{L}$ and $\mathrm{M}$. Therefore, we will take it to be a logical basis for MOA. ${ }^{14}$

Now, the MOA-structure can be presented as an arrangement $<\mathbf{T},(1), \alpha, X, \mathrm{~g}>$, where $\alpha$ is a sentence of the language of the (applied) modal logic including the sentence $\mathrm{g}$, and $X$ is a set of sentences resulting from the substitution of propositional variables by the sentence $g$ in the specific theses of modal propositional systems. The sentence $\alpha$ represents here a specific premise expressing the idea that the existence of God is not contingent, and the set $X$ represents the set of all general premises of the given MOA-version.

Since the elements $\mathbf{T},(1)$ and $g$ are fixed in every MOA-version, we will write it in a shortened form as " $[\alpha, X]$ ":

$$
[\alpha, X]=\langle\mathbf{T},(1), \alpha, X, \mathrm{~g}\rangle .
$$

By virtue of this convention, any version of the argument for philosophical theism applying the theses of the $\mathbf{T}$-system and having specific premises that include $\{(1)\}$ (non-modifiable) and $\{\alpha\} \cup X$ (modifiable) may be represented by the MOA-

\footnotetext{
11 Throughout the article, the bold symbols "T", "B" and "S5" stand for systems of modal logic, while the ordinary symbols "T", "B" and "S5" stand for sentences falling under axiom schemes appropriate to these systems.

12 Apart from specific and general premises, the laws of classical sentential logic are also applied in each version of the MOA (as in any other argument). This fact, which we take to be quite obvious, is not one of which we intend to make any special use.

13 The issue of the proper selection of general premises in the MOA was already raised in Kane (1984).

14 Similar assumptions can sometimes be found in literature on the MOA. Cf., for example, van Inwagen's remark that the formula ' $\mathrm{L} p \Rightarrow p$ ' "must be valid in every modal system in which the sentential operators represent possibility and necessity in any intuitive sense" (van Inwagen 2018, p. 242). Cf. also Eder and Ramharter (2015), where the authors state that the T-system would "seem to be mandatory on any modal conception of conceivability which can claim to be faithful to Anselm's reasoning" (p. 2814).
} 
structure $[\alpha, X]$. It is easy to see that the arrangement $[(2),\{\mathrm{T}\}]$ is an example of such MOA-structures, and that it is one that represents the T-version of the MOA.

The MOA-version (represented by the structure) $[\alpha, X]$ will be relevant if and only if $\alpha$ is a sentence clarifying the idea that the existence of God is not contingent, and $\alpha$ is logically equivalent (based on the $\mathbf{T}$-system) to a specific (i.e. non-general) sentence or conjunction of sentences with the following forms: ' $\beta \Rightarrow \mathrm{g}$ ', ' $\mathrm{L}(\beta \Rightarrow \mathrm{g})$ ', ' $\mathrm{g} \Rightarrow \beta$ ', or ' $\mathrm{L}(\mathrm{g} \Rightarrow \beta)$ '. The latter requirement's being fulfilled serves to reassure us that any clarification of the idea of God furnishes logically nontrivial, necessary and/or sufficient conditions for the existence of God. We embrace the idea that a formulation of such conditions will be methodologically fundamental where any philosophical theory of God is concerned.

The T-version $[(2),\{\mathrm{T}\}]$ is an example of the relevant MOA-versions. The following metatheorem testifies to this:

Metatheorem 1 Sentence (2) is logically equivalent (based on the T-system) to each of the following sentences:

(i) $(\mathrm{g} \Rightarrow \mathrm{Lg}) \wedge(\mathrm{Mg} \Rightarrow \mathrm{g})$,

(ii) $\sim(\mathrm{Mg} \wedge \mathrm{M} \sim \mathrm{g})$.

Proof Ad (i): For the implication from left to right, assume that $\sim \mathrm{Mg} \vee \operatorname{Lg}$. This sentence is equivalent to the sentence ' $\mathrm{Mg} \Rightarrow \mathrm{Lg}$ '. Hence, and from the two specific theses of the T-system ' $\mathrm{g} \Rightarrow \mathrm{Mg}$ ' and ' $\mathrm{Lg} \Rightarrow \mathrm{g}$ ', we get, by hypothetical syllogism, $\mathrm{g}$ $\Rightarrow \mathrm{Lg}$ and $\mathrm{Mg} \Rightarrow \mathrm{g}$. For the right-to-left implication, assume that $(\mathrm{g} \Rightarrow \mathrm{Lg}) \wedge(\mathrm{Mg} \Longrightarrow$ g). Again, by hypothetical syllogism, we get $\mathrm{Mg} \Rightarrow \mathrm{Lg}$. Ad (ii): based on de Morgan's law and the standard logical relations between modal operators. Q.E.D.

Sentence (i) of Metatheorem 1 specifies the necessary ( $g \Rightarrow \mathrm{Lg}$ ) and sufficient $(\mathrm{Mg} \Rightarrow \mathrm{g}$ ) conditions for God to exist. Sentence (ii), in turn, expresses the noncontingency of God's existence in the Aristotelian sense of bilateral possibility (cf. Łukasiewicz 1957).

Let us now consider the argument having the structure ['MLg', $\{\mathrm{B}\}]$, where $\mathrm{B}$ is the sentence that results when we substitute $\mathrm{g}$ for $p$ in the thesis 'ML $p \Rightarrow p$ ' of the B-system:

B

$\operatorname{MLg} \Rightarrow \mathrm{g}$.

This argument is an example of an MOA-version for which we do not see any possibility of demonstrating that it meets the criterion of relevance adopted. (We will leave open the matter of proving that this version does not really meet the relevance criterion). ${ }^{15}$

\footnotetext{
15 This MOA-version corresponds to the version considered in Oppy (1996) (cf. footnote 5).
} 


\section{Criteria of formal correctness and simplicity for modal ontological arguments}

In order to properly formulate a criterion of correctness for the MOA, we shall introduce the following definition:

Definition 1 If $\{\alpha\}, X$ are sets of sentences of the language of the T-system with the constant $\mathrm{g}$, then $X \vdash \alpha$ if and only if $\alpha$ is an element of the smallest set containing all substitutions of theses of the $\mathbf{T}$-system and elements of $X$ and closed under two inference rules - modus ponendo ponens and Gödel's Rule of Necessitation.

As usual, for short, we will write ' $\alpha_{1}, \ldots, \alpha_{n}, X \vdash \beta$ ' instead of ' $\left\{\alpha_{1}, \ldots, \alpha_{n},\right\} \cup X$ $\vdash \beta$ ', and ' $\vdash \beta$ ' instead of ' $\varnothing \vdash \beta$ '. We introduce the abbreviation:

$$
\mathbf{T G}(\alpha, X)=\{\beta:(1), \alpha, X \vdash \beta\} .
$$

We will, also for short, write 'TG( $(\alpha)$ ' instead of 'TG( $(\alpha, X)$ ', if $\mathbf{T G}(\alpha, X)=\mathbf{T G}(\alpha$, $\varnothing)$.

Thus, $\mathbf{T G}(\alpha, X)$ is the smallest set of sentences containing all substitutions of theses of the $\mathbf{T}$-system and elements of the set $\{(1), \alpha\} \cup X$, and closed under modus ponendo ponens and the Rule of Necessitation. In other words, $\mathbf{T G}(\alpha, X)$ is the theory resulting from the strengthening of $\mathbf{T}$ by adding axioms (1), $\alpha$ and all elements of $X$. Each such theory will be called a mini-theory of God. ${ }^{16}$

Intuitively, all elements of the set $\{(1), \alpha\} \cup X$ are a priori sentences; so if they are true, they are necessarily true. This assumption seems to be fully justified in the context of the considerations pertaining to MOA. It also justifies the above finding to the effect that the Rule of Necessitation applies not only to the laws of logic, but also to all of the elements of the set $\{(1), \alpha\} \cup X$. (This finding will allow us to simplify some of the proofs below.)

A MOA-version $[\alpha, X]$ will be non-circular if and only if the sentence $\mathrm{g}$ is not derived from $\{\alpha\} \cup X$ on the basis of the $\mathbf{T}$-system alone (and therefore is not derived from $\{\alpha\} \cup X$ independently of (1)); formally: $\alpha, X \not \forall$. ${ }^{17}$

The argument ['Lg', $\{\mathrm{T}\}]$ is an example of a valid but circular MOA-version. The fact that this argument can be treated as a relevant MOA-version at all is evidenced by the fact that the sentence ' $\mathrm{Lg}$ ' explicates the idea of the existence of God as a necessary existence. (More precisely, it states that God necessarily exists). It is valid and circular, because sentence $g$ is directly derivable from sentence ' $\mathrm{Lg}$ ' and premise $\mathrm{T}$-and thus is so regardless of (1).

As we can see, the concept of "validity" (used for a given MOA-version) has a broader extension than the concept of "formal correctness". Let us adopt the following definition:

\footnotetext{
16 The difference between objects $[\alpha, X]$ and $\mathbf{T G}(\alpha, X)$ is worth emphasizing. The former is the abstract structure of a given MOA-version, while the latter is a set of propositions underlying that version.

17 The formal concept of "circularity" used above differs from the intuitive concept of "begging the question" used by van Inwagen (2018).
} 
Definition 2 The MOA-version $[\alpha, X]$ will be formally correct if and only if the following three conditions are met:

(i) (1), $\alpha, X \vdash \mathrm{g}$ (VALIDITY),

(ii) $\mathbf{T G}(\alpha, X)$ is consistent (CONSISTENCY), and

(iii) $\quad \alpha, X \nvdash g$ (NON-CIRCULARITY).

Valid MOA-versions can be compared with respect to the number of general premises they possess and the deductive strengths of their own mini-theories. Let us adopt the following definition:

Definition $3[\alpha, X]$ will be a simpler MOA-version than $[\beta, Y]$ if and only if at least one of the following conditions is met (where $|X|$ is the cardinality of the set $X$ ):

(i) $|X|<|Y|$ and $\mathbf{T G}(\alpha, X) \subseteq \mathbf{T G}(\beta, Y)$, or

(ii) $|X| \leq|Y|$ and $\mathbf{T G}(\alpha, X) \subsetneq \mathbf{T G}(\beta, Y)$.

Definitions 1-3 will be deployed in due course - in the remainder of this paper.

\section{Formal correctness of three standard versions of the argument}

The T-version [(2), $\{\mathrm{T}\}]$ is an example of a relevant and formally correct MOAversion. Indeed, and especially given Metatheorem 1, the relevance of this version is obvious, and the following METATHEOREM holds true:

Metatheorem 2 The T-version of the MOA, represented by the structure [(2), $\{\mathrm{T}\}]$, is formally correct.

Proof The following three conditions must be shown to have been met:

(a) (1), (2), $\{\mathrm{T}\} \vdash \mathrm{g}$.

(b) $\mathbf{T G}(2)$ is consistent, and.

(c) (2), $\{\mathrm{T}\} \not \forall \mathrm{g}$.

Ad (a) The sentence $g$ was derived from the axioms of $\mathbf{T G}(2)$ in Sect. 1.

Ad (b) The language of the mini-theory $\mathbf{T G ( 2 )}$ can be formally interpreted as follows: the sentence $\mathrm{g}$ is interpreted as the constant 1 (true sentence), the operators $\mathrm{M}$ and $\mathrm{L}$ are interpreted as the assertion operator $\mathrm{A}$ (in the sense defined by the axiom 'A $\mathrm{A} \Leftrightarrow p$ '), and the truth-connectives are left unchanged. As a result, the set TG(2) becomes the theory $\mathbf{T G}(2)^{*}$, which contains only the constant 1 and the set of sentences resulting from the substitution of variables by this constant in tautologies of classical sentential logic (with the operator A). As we know, the set $\mathbf{T G}(2)^{*}$ is consistent; consequently, the set TG(2) is also consistent.

Ad (c) If $g$ were derived from TG(2), then $g$ would be true in any model in which TG(2) is true. To see that the opposite is the case, let us consider a TG $(2)^{\#}$-theory that will be the result of the following interpretation: $g$ is 
interpreted as the constant 0 (false sentence), the $\mathrm{M}$ and $\mathrm{L}$ operators are interpreted as the assertion operator $\mathrm{A}$, and the truth-connectives are left unchanged. The $\mathbf{T G}(2)^{\#}$-theory will therefore consist only of the relevant substitutions in respect of the tautologies. (More particularly, Axiom (2) ${ }^{\#}$ will be such a substitution, equivalent to the sentence ' $\sim 0 \vee 0$ '). At the same time, the sentence $\mathrm{g}^{\#}$ (the constant 0 ) is false. Q.E.D.

In the literature on the MOA, what is known as Anselm's Principle has tended to receive more frequent consideration than Principle (2):

Historically, the first formalized version of the MOA (originating from Hartshorne) should be represented by the structure [(3), $\{\mathrm{T}, \mathrm{N}, \mathrm{S} 5\}]$, where $\mathrm{N}$ is the result of substitution of $\mathrm{g}$ for $p$, and $\mathrm{Lg}$ for $q$, in the thesis ' $\mathrm{L}(p \Rightarrow q) \Rightarrow(\mathrm{M} p \Rightarrow$ $\mathrm{Mq}$ )' of $\mathbf{T}$ :

$\mathrm{N}$

$\mathrm{L}(\mathrm{g} \Rightarrow \mathrm{Lg}) \Rightarrow(\mathrm{Mg} \Rightarrow \mathrm{MLg})$

and S5 is the formula being a result of the similar substitution in the specific axiom of the modal system S5:

Metatheorem 3 The S5-version of the MOA, represented by the structure [(3), \{T, $\mathrm{N}, \mathrm{S} 5\}$ ], is formally correct.

Proof The parts of the proof relating to the conditions of consistency and noncircularity are analogous to the relevant parts of the proof of Metatheorem 2. It is therefore sufficient to show that the condition.

$$
\text { (1), (3), }\{\mathrm{T}, \mathrm{N}, \mathrm{S} 5\} \vdash \mathrm{g}
$$

is satisfied. By using the law of detachment (modus ponens) twice, we obtain the thesis ' $\mathrm{Mg} \Rightarrow \mathrm{MLg}$ ' (from (3) and $\mathrm{N}$ ), and then 'MLg' (by using the axiom (1)). Hence, from S5, we get the sentence 'Lg'. Hence, from T, we get the sentence $g$. Q. E.D.

The deductive basis of the S5-version can be simplified so as to arrive at the weaker mini-theory TG( $3,\{\mathrm{~N}, \mathrm{~B}\})$. This shows a key fragment of the proof of the following metatheorem:

Metatheorem 4 The B-version of the MOA, represented by the structure $[(3),\{\mathrm{N}$, $\mathrm{B}\}$ ], is formally correct. 
Proof The parts of the proof relating to the conditions of consistency and noncircularity are analogous to the relevant parts of the proof of Metatheorem 2. It is therefore sufficient to show that the condition.

$$
\text { (1), (3), }\{\mathrm{N}, \mathrm{B}\} \vdash \mathrm{g}
$$

is satisfied. By using $\mathrm{N}$ and the law of detachment, we obtain the thesis ' $\mathrm{Mg} \Rightarrow$ $\mathrm{MLg}$ ', and then 'MLg'. Hence, from B, we get the sentence $g$. Q.E.D. ${ }^{18}$

Using Definition 3, we can compare all of the above MOA-versions. The following metatheorem will be the result of such a comparison:

Metatheorem $5[(2),\{\mathrm{T}\}]$ is a simpler MOA-version than [(3), $\{\mathrm{T}, \mathrm{N}, \mathrm{S} 5\}]$ and $[(3),\{\mathrm{N}, \mathrm{B}\}]$.

Proof It is enough to note that two conditions are met: (i) $|\{\mathrm{T}\}|<|\{\mathrm{T}, \mathrm{N}, \mathrm{S} 5\}| \&$ TG (2) $\subseteq \mathbf{T G}(3,\{\mathrm{~S} 5\})$, and (ii) $|\{\mathrm{T}\}|<|\{\mathrm{N}, \mathrm{B}\}| \& \mathbf{T G}(2) \subseteq \mathbf{T G}(3,\{\mathrm{~B}\})$. Ad (i): Given that $\mathrm{T}$ is an S5-thesis, it is sufficient to show that (2) is a thesis of the mini-theory TG(3, $\{\mathrm{S} 5\})$. This becomes apparent when we consider that ' $\mathrm{Lg}$ ' is a thesis of this theory (see the penultimate step of Metatheorem 2). Hence, we may conclude that (2) is also a thesis of it. Ad (ii): Given that $\mathbf{T}$ is a B-thesis, it is sufficient to show that (2) is a thesis of the mini-theory $\mathbf{T G}(3,\{\mathrm{~B}\})$. If we consider Metatheorem 4, then we realize that the sentence $\mathrm{g}$ is a thesis of this theory. Eliminating operator $\mathrm{L}$ in formula (3) (according to the T-schema ' $\mathrm{L} \alpha \Rightarrow \alpha$ '), we get ' $\mathrm{g} \Rightarrow \mathrm{Lg}$ '. Hence, by modus ponens, we get $\mathrm{Lg}$. Thus, we may conclude that (2) is a thesis of the minitheory $\mathbf{T G}(3,\{B\})$. Q.E.D.

\section{The simplest relevant version of the modal ontological argument}

It will now be shown that there is a correct version of the MOA that is simpler than all the versions considered so far. ${ }^{19}$ The essence of this argument, which in logical terms borders on triviality, is the sentence.

(4) $\sim \mathrm{g} \Rightarrow \sim \mathrm{Mg}$

(if God does not exist, then God cannot exist).

\footnotetext{
${ }^{18}$ See Kane (1984, p. 339). According to Kane, this fact proves that the B-version is the right version of the MOA. Even so, the view that the S5-system is essential for a proper analysis of the MOA has been quite popular in the philosophical literature — cf., for example, this statement: "[...] all modal ontological arguments are valid in S5 (and they are valid in no weaker modal system [...])" (van Inwagen 2009, pp. 219-20).

${ }^{19}$ I shall make use of a similar result here to that arrived at in Biłat (2012a, b). (The latter being a slightly shortened translation of the former.).
} 
We shall demonstrate in what follows that this premise correctly (though not necessarily completely) clarifies the idea of the existence of God as a necessary existence.

Indeed, such a claim is indirectly evidenced by the fact that such a postulate has appeared in the works of Hartshorne and Plantinga. It was also clearly accepted by Malcolm:

What Anselm has proved is that the notion of contingent existence or of contingent nonexistence cannot have any application to God. His existence must either be logically necessary or logically impossible.[...] If God, a being a greater than which cannot be conceived, does not exist then He cannot come into existence.[...] Since He cannot come into existence, if He does not exist His existence is impossible. (Malcolm 1960, p. 49)

The direct justification for the thesis that (4) is the correct explication for the existence of God as a necessary existence runs in essence as follows: the idea is fully expressed (taking into account the object language of modal logic) in the form of Principle (2), and each of Postulates (3) and (4) can be treated as a part of the explication of the idea expressed by (2). Thus, if we assume that Principle (3) appropriately elucidates the aforementioned idea, then we should also assume that Principle (4) correctly explicates it. ${ }^{20}$

This explicative dependence of both postulates on (2) is quite clearly visible in the context of possible-worlds semantics. Both postulates are similarly derived from the ontological principle, relating to (2), that God exists either in every possible world (accessible from the actual world) or not in any of them. According to this principle, if God exists in the actual world, then God exists in every possible world which is the content of (3). ${ }^{21}$ Similarly, if God does not exist in the actual world, then God does not exist in any possible world-which in turn, is the content of (4).

The following metatheorem shows the logical connection of both postulates with (2):

Metatheorem $6 \vdash(2)$ if and only if $\vdash(3)$ and $\vdash(4)$.

Proof The proof here is similar to that of Metatheorem 1. For the implication from left to right, assume $\vdash$ ' $\sim \mathrm{Mg} \vee \mathrm{Lg}$ '. Hence, and from $\vdash$ ' $\mathrm{g} \Rightarrow \mathrm{Mg}$ ' and $\vdash$ ' $\mathrm{Lg} \Rightarrow \mathrm{g}$ ', we get $\vdash$ ' $\mathrm{g} \Rightarrow \mathrm{Lg}$ ' and (taking into consideration the Rule of Necessitation) $\vdash$ ' $\mathrm{L}$ (g $\Rightarrow \mathrm{Lg}) \wedge(\sim \mathrm{g} \Rightarrow \sim \mathrm{Mg})$ '. For the implication from right to left, assume $\vdash$ ' $\mathrm{L}(\mathrm{g} \Rightarrow$ $\mathrm{Lg}) \wedge(\sim \mathrm{g} \Rightarrow \sim \mathrm{Mg})$ '. We therefore get $\vdash$ ' $(\mathrm{g} \Rightarrow \mathrm{Lg}) \wedge(\mathrm{Mg} \Rightarrow \mathrm{g})$ ' and, by hypothetical syllogism: $\vdash$ ' $\mathrm{Mg} \Rightarrow \mathrm{Lg}$ '. Q.E.D.

It turns out that Premise (2) is logically equivalent to the conjunction of Premises (3) and (4).

\footnotetext{
${ }^{20}$ We omit here the fact that (3) is additionally preceded by a necessity operator. This is possible thanks to the assumption made here that Rule of Necessitation can also be applied to extra-logical a priori statements such as (4). (The modal status of (3) and (4), as a priori statements, is exactly the same).

21 This principle can also be expressed in Plantinga's style: if God exists in the actual world, then God is maximally great (cf. Plantinga 1974).
} 
Moreover, (4) is deductively weaker than both (2) and (3), where this stated by another metatheorem:

\section{Metatheorem 7 The following relations hold:}

(i) (2) $\vdash(4)$,

(ii) $\quad(3),\{\mathrm{N}, \mathrm{B}\} \vdash(4)$,

(iii) (4) $\forall(2)$,

(iv) (4) $\nvdash(3)$.

Proof Ad (i): obvious. Ad (ii): as with the proof of Metatheorem 4. Ad (iii) and (iv): it is enough to consider the possible-worlds model in which $g$ is true in the actual world and there are possible worlds accessible from the actual world in which $\mathrm{g}$ is not true; we see that in this model (4) is true and (2) and (3) are not true. Q.E.D.

Consequently, since (3) means that the existence of God is either impossible or necessary, (2) and (3) only partially clarify this meaning. In fact, (3) only means that if God exists, he exists by necessity, and (4) only means that if God does not exist, he does not exist by necessity. Only both sentences taken together fully express the idea of God as a non-contingent being. ${ }^{22}$

Thus, from an ontological and a logical point of view, Premise (4) is not less obvious, more controversial or in any other sense stronger than (3). On the contrary, taking into account conditions (ii) and (iv) of Metatheorem 7, Premise (4) is deductively weaker than (3). If we accept (3) as an intuitively acceptable premise in one or other of the MOA-versions, we must surely proceed likewise with (4). Premise (3) is commonly treated as being the result of a typical explication of the basic idea of God, so there is no reason for Premise (4) to be treated any differently. $^{23}$

We sometimes encounter sentence (4), or its logical equivalents, being treated as premises for a complex MOA-version, in which they are further formally justified (see, e.g., Spencer 2018, p. 214). However, it appears that the persuasive power of (4) is no less than that of similar premises such as are normally accepted without any formal justification. Thus, (4) does not call for such justification more than in the case of other MOA-versions.

Since Postulate (4) explicates the idea of the existence of God as a necessary existence, the mini-theory $\mathbf{T G}((4), \varnothing)$ represents one of the MOA-versions meeting the relevance condition.

Metatheorem 8 The “empty" structure [(4), Ø] represents a formally correct MOA-version which is simpler than T-version.

Proof The parts of the proof relating to the conditions of consistency and noncircularity are analogous to the relevant parts of the proof of Metatheorem 2. It is

\footnotetext{
22 This topic of the explicative dependencies, respectively, of (3) and (4) on (2), will be developed in the next section.

23 Postulate (4) is logically equivalent to the thesis ' $\mathrm{Mg} \Rightarrow \mathrm{g}$ '. This MOA-version is even closer to Leibniz's conception of God (defined as the necessarily existing being), according to which if God is possible, then God exists (see Griffin 2012, pp. 42, 43; Antognazza 2018, pp. 75, 78-89).
} 
therefore enough to note that $|\varnothing|<|\{T\}|$, and the sentence $g$ is a thesis of the minitheory TG(2) (as we know from Metatheorem 2). Therefore, it is all the more the case that (4) is one of its theses. Q.E.D.

\section{Some stronger correctness and relevance criteria for the modal ontological argument}

The logical triviality of the "empty" MOA-version suggests that the range of formally correct and philosophically relevant MOA-versions should be limited in such a way that this version can be considered incorrect or irrelevant. Consider the two options outlined below.

It seems that the only correspondingly appropriate way to limit the concept of formal correctness is to introduce an additional condition into Definition 2:

(C)

$\mathrm{g}, X \nvdash \alpha$.

This condition states (in simple terms) that the specific premise that defines the concept of God as a non-contingent being cannot be a consequence of the thesis that God exists. At first glance, the following metatheorem would seem to support a possible decision to introduce this condition:

Metatheorem 9 If $[\alpha, X]$ is valid and $\mathrm{g}, X \vdash \alpha$, then $X \vdash ' \mathrm{~g} \Leftrightarrow(\operatorname{Mg} \wedge \alpha)$ '.

Proof The second part of the antecedent of the metatheorem is equivalent (in virtue of the deduction theorem) to the meta-formula $X \vdash{ }^{\prime} \mathrm{g} \Rightarrow \alpha$ '. Hence, taking into consideration that $X \vdash$ ' $\mathrm{g} \Rightarrow \mathrm{Mg}$ ', we get: $X \vdash$ ' $\mathrm{g} \Rightarrow(\mathrm{Mg} \wedge \alpha)$ '. Since $[\alpha, X]$ is valid, the reverse implication is also derivable: $X \vdash{ }^{\prime}(\operatorname{Mg} \wedge \alpha) \Rightarrow$ g'. Q.E.D.

Metatheorem 9 shows that any valid MOA-version that does not meet (C) is "circular" in the sense that the conjunction of its premises is logically equivalent to its conclusion. (We are, at the very least, using the term "logically" here just as it pertains to modal logic).

The following metatheorem shows that the "empty" MOA-version does not meet Condition (C):

\section{Metatheorem $10 \mathrm{~g} \vdash(4)$.}

Proof If we take into account the Duns Scotus Law, we realize that $\vdash$ ' $g \Rightarrow(\sim g$ $\Rightarrow \sim \mathrm{Mg})$ '. Keeping in mind the deductive theorem, we thus get: $\mathrm{g} \vdash^{\prime} \sim \mathrm{g} \Rightarrow \sim$ Mg'. Q.E.D.

Unlike (4), Premises (2) and (3) cannot be derived from Sentence g alone, together with the laws of modal logic. (Let us leave this observation without proof). This shows that Condition (C) could be used to eliminate the "empty" MOAversion. 
The question is whether Condition (C) should be accepted. Let us recall its general content: the premise defining the concept of God cannot be a consequence of the thesis that God exists. But why not? There is no logical, methodological or philosophical reason to accept such a restriction. Apparently, an attempt to introduce it would be an ad hoc solution, only aimed at eliminating one of the MOA-versions.

Let us therefore consider the second option (referred to at the beginning of this section). A quite natural way of strengthening the relevance condition was already indicated in Metatheorems 1 and 6, and in the analysis of the previous section. Its philosophical basis runs as follows: an MOA-version will be strongly relevant if the premise defining the philosophical concept of God (as a non-contingent being) is a complete explication of that concept. According to Metatheorems 1 and 6, and the analyses carried out in the previous section, Postulate (2) is such a complete explication, as opposed to Postulates (3) and (4). Consequently, the T-version is, in contrast to other versions, a strongly relevant MOA-version.

Let us recall the general distinction between the two types of MOA-relevance, and try to find a good philosophical basis for it. Postulate (2) is a complete explication of the idea of God as a non-contingent being in the language of the minitheory TG(2). Postulate (3) is its partial explication, because (3) only expresses a necessary condition for the existence of God. Postulate (4) is also its partial explication, but for another reason: because (4) only expresses a sufficient condition for this existence. Unlike mini-theories TG(3, $\{$ S5 $\}), \mathbf{T G}(3,\{\mathrm{~B}\})$ and $\mathbf{T G}(4)$, the mini-theory TG(2) generates both a necessary and a sufficient condition for God to exist. So, from a philosophical and theoretical point of view, TG(2) is a better minitheory than the others and, consequently, the T-version is a better MOA-version than the others.

This does not mean that the MOA-version represented by the structure $[(2),\{\mathrm{T}\}]$ is absolutely preferable to the structures [(3), $\{\mathrm{T}, \mathrm{N}, \mathrm{S} 5\}],[(3),\{\mathrm{N}, \mathrm{B}\}]$ and [(4), Ø]. On the contrary, according to the general theory of argumentation, there are many types of relevance depending on kinds of arguments and their conversational contexts (see, e.g., Walton 1998). In the case of a philosophical argument, its context can be determined equally by its persuasive and explicative (or, more precisely, theoretical-explicative) purpose. If the argument is formulated in a persuasive context, the requirement of full explication of the notions used in it, and therefore the requirement of strong relevance, does not apply.

Consequently, we should use two criteria in assessing MOA-versions: the weak criterion and the strong criterion of MOA-relevance. If the MOA-version is formulated to convince someone that the God of philosophers exists, the strong criterion is unnecessary. This criterion, on the other hand, is essential for the evaluation of each MOA-version formulated in order to examine the consequences of the explicatively complete concept of God.

Given the persuasive function of the argument, the "empty" MOA-version would seem to be the optimal one. There are at least two reasons for this assessment. Firstly, the "empty" version is the simplest of the formally correct and (weakly) relevant MOA-versions (cf. Metatheorems 5-7). Secondly, the "empty" version mounts an effective defence against a typical counter-argument that purports to 
show the persuasive weakness of the standard MOA-versions. Let us replace Premise (1) with the sentence ' $\mathrm{M} \sim \mathrm{g}$ ' ("It is possible that God does not exist"); hence, from (2) we derive (in the T-system) ' g' ("God does not exist"), and from (3) we also therefore derive (in the S5-system) ' g' (cf. Oppy 1996, 2019). It is easy to see that there is no analogous counter-argument to the "empty" MOAversion.

\section{Conclusions}

The questions posed at the end of the first section can now be answered quite precisely. Each relevant (zero-order, Hartshorne-style) MOA-version has the structure $<\mathbf{T}$, (1), $\alpha, X, \mathrm{~g}>$, where $\alpha$ is a specific premise clarifying the idea that the existence of God is not contingent and $X$ is a set of general premises resulting from modal logic. The formal correctness criterion for such versions consists of conditions of VALIDITY, CONSISTENCY, and NON-CIRCULARITY.

It turns out that the simplest known MOA-version fulfilling these conditions has the structure [(4), Ø]. In contrast to the previously presented versions (T, S5 and B), this "empty" MOA-version is devoid of general premises taken from modal logic. Thus, its entire strength lies in its specific philosophical premises, not in its logic. (These premises state that the existence of God is possible, and that if God does not exist, the existence of God is impossible.)

Given the persuasive function of the argument, the "empty" version seems to be the basic MOA-version on account of its simplicity (consisting in its formal simplicity and the deductive weakness of its mini-theory of God) and its resistance to a well-known counter-argument from the possibility of the non-existence of God.

Of all the MOA-versions considered here, only the T-version meets the strong relevance condition of explicative completeness, because only this version expresses precisely the idea (from Aristotle) of God's non-contingency in the form of a necessary and a sufficient condition for the existence of God. For this reason, from a theoretical point of view (although not necessarily from a persuasive point of view), the T-version should be treated as the basic MOA-version.

These conclusions undermine the view (mentioned in Sect. 1) that certain general premises generated by stronger systems of modal logic are logically essential, or at least highly adequate philosophically, where the MOA is concerned. Let us recall both of the theses suggested by van Inwagen:

Thesis 1 Each formally correct and relevant MOA-version has either strong specific premises and weak general premises (van Inwagen 2012, p. 158, is referring here to the T-version) or, equally, weak specific premises and strong general premises (he is referring here to the S5-version).

Thesis 2 The option indicated in the second part of this equivalence (contained in Thesis 1) is philosophically better. 
Both theses turn out to be false, assuming the criteria adopted here for the evaluation and selection of MOA-versions. $^{24}$

Firstly, there is a formally correct and persuasively relevant MOA-version (namely, the "empty" version) that has relatively weak specific (metaphysical) premises and no general premises. Thus, van Inwagen's specification omits the type of MOA-version that plays a key role in our analysis. Moreover, the equivalence contained in Thesis 1 is incorrect in one important respect: the T-version (indicated on the left) is not philosophically equivalent to the S5-version (indicated on the right). The S5-version, unlike the T-version, is based on an explicatively incomplete mini-theory of God. (More precisely, the mini-theory of God underlying the S5version generates a necessary condition for the existence of God, but does not generate a sufficient condition for it.)

Secondly, both because of this explicative incompleteness and on account of its complexity, the S5-version is philosophically inferior to the T-version. Moreover, despite the lesser complexity of the B-version (relative to the S5-version), that same conclusion applies to it, too.

Open Access This article is licensed under a Creative Commons Attribution 4.0 International License, which permits use, sharing, adaptation, distribution and reproduction in any medium or format, as long as you give appropriate credit to the original author(s) and the source, provide a link to the Creative Commons licence, and indicate if changes were made. The images or other third party material in this article are included in the article's Creative Commons licence, unless indicated otherwise in a credit line to the material. If material is not included in the article's Creative Commons licence and your intended use is not permitted by statutory regulation or exceeds the permitted use, you will need to obtain permission directly from the copyright holder. To view a copy of this licence, visit http:// creativecommons.org/licenses/by/4.0/.

\section{References}

Antognazza, M. R. (2018). Leibniz. In G. Oppy (Ed.), Ontological Arguments (pp. 75-98). Cambridge: Cambridge University Press.

Benzmüller, C. (2020). A (simplified) supreme being necessarily exists, says the computer: Computationally explored variants of Gödel's ontological argument. Cornell University. https://arxiv.org/abs/ 2001.04701

Biłat, A. (2012a). Dowód ontologiczny a logika modalna. Filozofia Nauki, XX(1(77)), 103-108. ((in Polish)).

Biłat, A. (2012b). Modal logic vs. ontological argument. European Journal for Philosophy of Religion, 4 (2), 179-185.

Blackburn, S. (1994). The Oxford dictionary of philosophy. Oxford: Oxford University Press.

Chellas, B. F. (1980). Modal logic. An introduction. Cambridge: Cambridge University Press.

Chellas, B. F., \& Segerberg, K. (1994). Modal logic with the MacIntosh rule. Journal of Philosophical Logic, 23(1), 67-86.

Eder, G., \& Ramharter, E. (2015). Formal reconstructions of St. Anselm's ontological argument. Synthese, 192(9), 2795-2825. https://doi.org/10.1007/s11229-015-0682-8

Goodwin, G. L. (1978). The ontological argument of Charles Hartshorne. Missoula: Montana Scholars Press.

Griffin, M. (2012). The ontological argument, the principle of sufficient reason and Leibniz's doctrine of striving possibles. In M. Griffin (Ed.), Leibniz, God and necessity (pp. 34-57). Cambridge: Cambridge University Press. https://doi.org/10.1017/CBO9781139022286.003.

\footnotetext{
${ }^{24}$ It is worth noting that Theses 1 and 2 do not perform any key role as regards the main theses of van Inwagen's article quoted above.
} 
Hartshorne, C. (1944). The formal validity and the real significance of the ontological argument. The Philosophical Review, 53(3), 225-245.

Kane, R. (1984). The modal ontological argument. Mind, 93(371), 336-350.

Lewis, D. (1970). Anselm and actuality. Noûs, 4, 175-188.

Łukasiewicz, J. (1957). Aristotle's syllogistic from the standpoint of modern formal logic. Oxford: Clarendon Press.

Malcolm, N. (1960). Anselm's ontological arguments. The Philosophical Review, 69(1), 41-62.

Oppy, G. (1996). Ontological arguments and belief in God. Cambridge: Cambridge University Press.

Oppy, G. (2019). Ontological arguments. In E. N. Zalta (Ed.), The Stanford encyclopedia of philosophy. Stanford: Stanford University.

Perzanowski, J. (1991). Ontological arguments II: Cartesian and Leibnizian. In H. Burkhardt \& B. Smith (Eds.), Handbook of metaphysics and philosophy (pp. 625-633). Munich: Philosophia Verlag.

Plantinga, A. (1974). The nature of necessity. Oxford: Oxford University Press.

Sobel, J. H. (1987). Gödel's ontological proof. In J. J. Thompson (Ed.), On being and Saying: Essays for Richard Cartwright (pp. 241-261). Cambridge: MIT Press.

Spencer, J. (2018). Conceivability and possibility. In G. Oppy (Ed.), Ontological arguments (pp. 214237). Cambridge: Cambridge University Press.

Świętorzecka, K., \& Łyczak, M. (2018). An even more Leibnizian version of Gödel's ontological argument. Journal of Applied Logic, 5(7), 1553-1566.

van Inwagen, P. (2009). Some remarks on the modal ontological argument. Philo, 12(2), 217-227.

van Inwagen, P. (2012). Three versions of the ontological argument. In M. Szatkowski (Ed.), Ontological proofs today (pp. 143-162). Heusenstamm: Ontos Verlag.

van Inwagen, P. (2018). Begging the question. In G. Oppy (Ed.), Ontological arguments (pp. 238-249). Cambridge: Cambridge University Press.

Walton, D. N. (1998). The new dialectic: Conversational contexts of argument. Toronto: University of Toronto Press.

Publisher's Note Springer Nature remains neutral with regard to jurisdictional claims in published maps and institutional affiliations. 